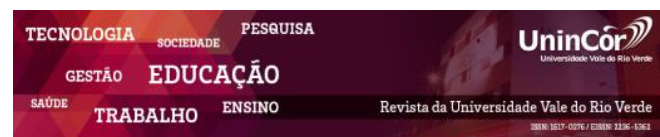

Revista da Universidade Vale do Rio Verde ISSN: 1517-0276 / EISSN: 2236-5362 v. $17 \mid$ n. 1 | Ano 2019

Adelar Francisco Baggio Doutorando do Programa de Pós-Graduação em Desenvolvimento Regional /UNIJUI baggio@unijui.edu.br

Daniel Knebel Baggio Professor do Programa de Pós-Graduação em Desenvolvimento Regional - PGDR/UNIJUI e do Programa de Pós-Graduação em Gestão Estratégica de Organizações - PPGGEO/URI danibaggio@gmail.com

Martinho Luis Kelm Professor do Programa de Pós-Graduação em Desenvolvimento Regional - PGDR/UNIJUI martinho@unijui.edu.br

\section{DESAFIOS, EXPERIÊNCIAS E ESTRATÉGIAS DE ADOÇÃO DA GOVERNANÇA EM COOPERATIVAS}

\section{RESUMO}

$\mathrm{O}$ presente artigo tem como objetivo principal identificar as principais preocupações dos dirigentes de cooperativas com relação à governança e propor contribuições que possam servir de orientações para acelerar sua adoção nas respectivas organizações. Refere-se a um estudo qualitativo e descritivo. A coleta de dados ocorreu junto aos dirigentes de cinco cooperativas localizadas no Rio Grande do Sul, a partir de entrevistas semi-estruturadas. $\mathrm{O}$ diagnóstico expresso no trabalho disponibiliza valiosos subsídios que poderão servir de referência ou ponto de partida para estudos mais aprofundados e formulação de estratégias visando a implantação da governança nas cooperativas. De forma resumida, pode-se afirmar que não existe uma compreensão consolidada sobre governança nas cooperativas e que, ainda, não existe uma disposição fortemente deliberada para a sua adoção. Portanto, sugere-se que haja maior estímulo e divulgação por parte do Sistema Cooperativista e das Universidades junto aos dirigentes de cooperativas, assegurando, assim, a motivação e a coragem que viabilizem sua adoção, oportunizando melhores resultados para os cooperados e a comunidade das suas respectivas regiões.

Palavras-chave: Governança. Cooperativas. Cooperativismo. Governança Cooperativa. Governança Corporativa.

\section{CHALLENGES, EXPERIENCES AND STRATEGIES FOR THE ADOPTION OF GOVERNANCE IN COOPERATIVES}

\begin{abstract}
The objective of this article is to identify the main concerns of cooperative leaders regarding governance and to propose contributions that could serve as guidelines to accelerate their adoption in their respective organizations. Refers to a qualitative and descriptive study. Data were collected from the managers of five cooperatives located in Rio Grande do Sul, based on semistructured interviews. The diagnosis expressed in the work provides valuable subsidies that can serve as a reference or starting point for further studies and formulation of strategies for the implementation of cooperative governance. In summary, it can be said that there is no consolidated understanding of governance in cooperatives, and that there is no strong deliberate provision for its adoption. Therefore, it is suggested that there is a greater stimulus and dissemination by the Cooperative System and the Universities to the leaders of cooperatives, thus ensuring the motivation and courage that will enable its adoption, providing better results for the cooperative and the community of their regions.
\end{abstract}


Keywords: Governance. Cooperatives. Cooperativism. Cooperative Governance. Corporate Governance.

Recebido em: 04/10/2018 - Aprovado em: 21/03/2019 - Disponibilizado em: 15/07/2019

\section{INTRODUÇÂO}

As cooperativas, no decorrer da história, se apropriam de teorias e técnicas da administração de organizações para uso na gestão dos seus empreendimentos. Contudo, a adoção destas teorias e técnicas normalmente ocorre de forma tardia, em comparação com as organizações mercantis. O mesmo ocorreu, com relação à governança, pois apenas recentemente as cooperativas estão buscando adotar a governança após décadas de uso pelas empresas mercantis, de forma especial, pelas corporações que negociam suas ações nas Bolsas de Valores nos diferentes países.

Neste sentido, o presente artigo tem como objetivo principal identificar as principais preocupações dos dirigentes de cooperativas com relação à governança e propor algumas contribuições que podem servir de orientações para acelerar sua adoção nas respectivas organizações. O estudo buscou identificar as "vozes e sentimentos" vivenciados no dia-a-dia e propor algumas contribuições que podem servir como diretrizes visando acelerar a adoção da governança. Portanto, o texto foi elaborado após ouvir uma dezena de presidentes e executivos de cooperativas que integram os Ramos Agropecuário, Saúde, Infraestrutura e Crédito.

\section{COMPREENDEDO A GOVERNANÇA}

São quatro os conceitos de governança voltados para as cooperativas:

a) Do Instituto Brasileiro de Governança Corporativa-IBGC (2015, p.13): Governança Corporativa, "é o sistema pelo qual as organizações são dirigidas, monitoradas e incentivadas, envolvendo os relacionamentos entre proprietários/cooperados, conselho de administração, diretoria e órgãos de controle (conselho fiscal, auditorias e outros)";

b) Do Sistema OCB (CNCOOP-OCBSESCOOP): "Trata-se de um modelo de direção estratégica, fundamentado nos valores e princípios cooperativistas, que estabelece práticas éticas visando garantir a consecução dos objetivos sociais e assegurar a gestão da cooperativa de modo sustentável em consonância com os interesses dos cooperados" (OCB, 2015, p. 13);

c) Do BANCO CENTRAL DO BRASIL (2008, s/p): “Governança Cooperativa é o conjunto de mecanismos e controles, internos e externos, que permite aos cooperados definir e assegurar a execução dos objetivos da cooperativa, garantindo sua continuidade e os princípios cooperativistas". 


\section{GOVERNANÇA EM COOPERATIVAS}

Existe farta produção intelectual relacionada com a governança corporativa e não se pode dizer o mesmo sobre a produção voltada para a governança cooperativa. Isto representa um grande desafio e uma valiosa oportunidade para os Programas de Pós-Graduação e para os pesquisadores brasileiros.

Os documentos básicos oficiais que estão disponíveis sobre governança cooperativa no Brasil são:

1) "Diretrizes para Boas Práticas de Governança em Cooperativas de Crédito" e "Diretrizes e Mecanismos para Fortalecimento da Governança em Cooperativas de Crédito," publicados pelo Banco Central do Brasil em 2008;

2) Guia das Melhores Práticas de Governança para Cooperativas, publicado pelo IBGC em 2015;

3) Manual de Boas Práticas de Governança Cooperativa publicada pelo Sistema OCB em 2013;

4) Compêndios de Boas Práticas de Gestão e Governança em Cooperativas, publicados anualmente pelo Serviço Nacional de Aprendizagem do Cooperativismo SESCOOP em conjunto com a Fundação Nacional da Qualidade-FNQ a partir do Ciclo 2013/2014.

As cooperativas que iniciaram a implantação da governança antes de 2013 buscaram subsídios técnicos de forma mais intensa no IBGC, em consultorias especializadas, nos parâmetros da Organização para a Cooperação e para o Desenvolvimento Econômico
(OCDE) e através do benchmarking com organizações nacionais e internacionais que já tinham adotado a governança no seu processo de gestão, de forma mais acentuado com aquelas que integram as listas de corporações das diferentes Bolsas de Valores.

\section{METODOLOGIA}

A coleta de dados junto aos dirigentes de cooperativas, sujeitos da pesquisa, ocorreu através de entrevistas semiestruturadas. As informações obtidas junto aos entrevistados foram sistematizadas com base nas temáticas que integravam o referido roteiro e transcritas de tal forma que assegurassem a fidelidade das manifestações dos dirigentes. Os resultados buscados pelo estudo visavam identificar posicionamentos específicos de dirigentes de cooperativas percebidos e vivenciados no dia-adia da gestão de suas cooperativas e dos diálogos que ocorrem normalmente entre eles.

\section{ADOÇÃO DA GOVERNANÇA NAS COOPERATIVAS}

Certamente, existem razões que motivam as Diretorias e os Conselhos de Administração para adotarem a governança nas cooperativas, como existem razões que justificam a não adoção.

Os principais motivos apresentados pelos dirigentes de cooperativas contrários à sua adoção são: a) Perda de poder, pois defendem o estilo presidencialista de gestão, que acumula o papel político e executivo numa mesma pessoa. Compreendem que o "executivo" vai ter mais poder do que o Presidente que foi eleito pela assembleia ou pelo Conselho de Administração da respectiva cooperativa; b) A nova fórmula pode 
trazer riscos para a cooperativa, pois depende muito da capacidade, do desempenho e do comprometimento do executivo contratado; c) As eventuais consequências negativas da gestão da cooperativa serão de responsabilidade do Presidente; d) A Cooperativa, afirmam a Diretoria Eleita e/ou Conselho de Administração, há anos, utiliza um modelo que está dando certo, portanto não há necessidade de mudar; e) A equipe técnica da Diretoria se manifesta contraria a adoção da Governança; f) Não existe conhecimento adequado por parte da cooperativa sobre Governança; g) O Conselho de Administração entende que a cooperativa não está "madura" para tal; h) O Conselho não tem disponível uma pessoa que pode ser o executivo contratado; i) Não existe visão clara e abrangente do que representa a dimensão societária da cooperativa, bem como da dimensão social da mesma.

Uma das principais causas da resistência da adoção da governança nas cooperativas relaciona-se com a cultura existente no Brasil, pois segundo Bialoskorski Neto (2012, p. 98) “o presidente-membro de cooperativas brasileiras é frequentemente o CEO da cooperativa, principal agente das mesmas relações contratuais, acumula na maioria das vezes a presidência do conselho, a presidência das organizações, e é o principal diretor executivo. Esta situação pode estar associada com a lei brasileira que coloca a responsabilidade dos membros do conselho e o presidente da cooperativa".

Quanto aos fatores que facilitam a adoção segundo os mesmos dirigentes são: a) Conhecimento e atitude favorável do Presidente; b) Postura do Conselho de Administração favorável ao processo de adoção; c) Benchmarking executado junto a outras organizações nacionais e estrangeiras; d) Exigências impostas de parceiros comerciais da cooperativa; e) Consciência da importância do caráter social da cooperativa e dos princípios da governança; f) Conhecimento das vantagens da Governança em termos da profissionalização da gestão, de melhor desempenho e da longevidade organizacional; g) Exigências impostas de órgãos governamentais, tais como Banco Central, Agências Nacionais, entre outros; h) A governança oportuniza ao Presidente e ao Conselho acompanhar e supervisionar de perto a gestão da cooperativa, portanto facilita a participação, a transparência e o comprometimento dos dirigentes eleitos e dos associados; i) $\mathrm{O}$ interesse de Presidentes que ocuparam o cargo por muitos anos e percebem que podem "prolongar seu emprego" na cooperativa como executivos; j) Oportuniza maior estabilidade à cooperativa na troca da Diretoria e de membros do Conselho de Administração; k) O Presidente tem melhores condições de tratar das questões societárias da organização e de participar da Governança Territorial da área de atuação da respectiva cooperativa.

Deve-se destacar, ainda, que os dirigentes entrevistados indicaram os seguintes agentes como sendo cruciais para a adoção da governança: o presidente, o conselho de administração e a escolha da metodologia adequada, com assessoria especializada. 


\section{OS PRINCÍPIOS BÁSCIOS DA GOVERNANÇA}

O quadro 1 evidencia os princípios da governança corporativa e cooperativa definidos pelo IBGC e pelo Sistema OCB.

Quadro 1 - Princípios da Governança Corporativa e Cooperativa

\begin{tabular}{|c|c|c|c|}
\hline \multicolumn{3}{|c|}{ PRINCÍPIOS DA GOVERNANÇA } & \multirow{2}{*}{$\begin{array}{l}\text { PRINCÍPIOS DO } \\
\text { COOPERATIVISMO }\end{array}$} \\
\hline \multirow[t]{2}{*}{ CORPORATIVA } & \multicolumn{2}{|c|}{ COOPERATIVA } & \\
\hline & IBGC & ОСВ & \\
\hline $\begin{array}{l}\text { Senso de Justiça } \\
\text { e Equidade } \\
\text { (Fairness ou Equilty) }\end{array}$ & Transparência & Transparência & $\begin{array}{l}\text { Gestão Democrática Adesão } \\
\text { Voluntária e livre }\end{array}$ \\
\hline $\begin{array}{l}\text { Transparência } \\
\text { (Disclosure) }\end{array}$ & Equidade & Senso de Justiça & $\begin{array}{l}\text { Participação econômica dos } \\
\text { membros }\end{array}$ \\
\hline $\begin{array}{l}\text { Prestação Responsável } \\
\text { de Contas } \\
\text { (Accountability) }\end{array}$ & $\begin{array}{l}\text { Prestação de } \\
\text { Contas }\end{array}$ & Autogestão & Autonomia e Independência \\
\hline \multirow{3}{*}{$\begin{array}{l}\text { Responsabilidade } \\
\text { Corporativa } \\
\text { (Compliance) }\end{array}$} & $\begin{array}{l}\text { Responsabilidad } \\
\text { e Corporativa }\end{array}$ & Sustentabilidade & $\begin{array}{l}\text { Educação, formação e } \\
\text { informação }\end{array}$ \\
\hline & & Educação & Itercooperação \\
\hline & $\begin{array}{l}\text { (Mais os } \\
\text { Princípios do } \\
\text { Cooperativismo) }\end{array}$ & $\begin{array}{l}\text { (Mais os } \\
\text { Princípios do } \\
\text { Cooperativismo) }\end{array}$ & Interesse pela Comunidade \\
\hline
\end{tabular}

Fonte: Sistema OCB, 2015, p. 8-10; IBGC, 2016, p.15-16 e OCB, 2015, p.14

O quadro 1 evidencia que os princípios da governança corporativa são os clássicos mundialmente aceitos e os princípios da governança cooperativa têm duas versões distintas, uma definida pela OCB e outra pelo IBGC.

Outros referenciais utilizados pelas cooperativas são os princípios que integram o Código das Boas Práticas de Governança da OCDE citados pelo Rossetti (2014, p. 186), quais sejam:1) Garantir a base para uma eficaz governança corporativa; 2) Direitos dos acionistas e principais funções da propriedade; 3) Tratamento equitativo dos acionistas; 4) Papel de outras partes interessadas na governança corporativa; 5) Divulgação e transparência; 6) Responsabilidades do Conselho de Administração.

\section{ESTRATÉGIAS UTILIZADAS PELAS} COOPERATIVAS NA ADOÇÃO DA GOVERNANÇA

As estratégias facilitadoras identificadas junto às cooperativas que já adotaram a governança são as seguintes:

a) Tomar em consideração os referenciais da governança corporativa; b) Buscar referências nas cooperativas de crédito, nos documentos do Banco Central, nos documentos do IBGC, nos documentos do Sistema OCB, nos parâmetros da OCDE, e em subsídios disponibilizados pelo cooperativismo da Europa, no benchmarking junto a organizações nacionais e internacionais (mercantis e/ou não mercantis); c) Assessorias externas especializadas; d) Qualificação e/ou participação de pessoas 
da cooperativa em eventos técnicos voltados para a governança; e) Estímulos do Sistema Cooperativista Brasileiro através da OCB, das organizações estaduais e das entidades nacionais e estaduais dos respectivos Ramos Cooperativistas; f) Imposição de órgãos externos governamentais ou comerciais às cooperativas, tais como bancos, legislação, agências nacionais, etc.; g) Sinalização dos Bancos para as cooperativas obterem financiamentos; h) Presidentes que estão no cargo há anos e que desejam continuar como dirigentes, contudo não mais como presidentes, mas como executivos contratados; i) Imitar outras cooperativas que decidiram implantar a governança, embora as mudanças se tenham limitado apenas em ajustes nos Estatutos e Regimentos.

\section{ESTRANGULAMENTOS}

Os principais entraves que estão dificultando a adoção da governança em cooperativas segundo os dirigentes entrevistados são:

a) Pouco entendimento por parte da Diretoria e do Conselho de Administração sobre a temática; b) Resistência às inovações; c) Pouca clareza sobre o que significa a dimensão social e a dimensão econômica da cooperativa; d) Dúvidas com relação ao papel dos funcionários na "sociedade cooperativa" no contexto do novo modelo de gestão; e) Dúvidas sobre o que significa o princípio cooperativista "interesse pela comunidade;" f) Resistências na se- gregação do papel do presidente e do executivo contratado; g) Capacidade não adequada do presidente para a gestão dos conflitos de agência e da construção de convergências estratégicas entre os dirigentes eleitos e os executivos contratados; h) Dificuldades na construção dos interesses convergentes entre todos os agentes da governança; i) Resistência com relação à organização e estruturação do quadro social e de institucionalizar mecanismos de participação dos associados na governança; j) Dificuldades na implantação e na gestão de sistemas de controle e avaliação dos executivos contratados.

\section{SINGULARIDADE COOPERATIVAS}

DAS

Pode-se destacar as seguintes características que diferenciam as cooperativas das demais organizações e que devem ser consideradas no momento da adoção da governança: a) Sociedade de pessoas e não de capital; b) Possui princípios doutrinários específicos definidos pelo cooperativismo; c) Cada associado um voto; d) Associado é ao mesmo tempo dono e usuário da cooperativa; e) Não visa lucro, mas atendimento às necessidades comuns dos proprietários; f) Pode haver diferentes interesses entre os dirigentes eleitos, os gestores e os associados; g) São os associados que escolhem os gestores da Cooperativa; h) As cooperativas podem criar mecanismos específicos que oportunizem participação dos associados na gestão da cooperativa; i) As cooperativas têm a possibilidade de construir o coletivo dos 
associados, relativizando o individualismo e a concessão de privilégios especiais; j) Diversidade e conflitos de interesses entre os associados ou entre grupos de cooperados; k) Embora sejam administradas por associados pode emergir problemas de agência e de direitos de propriedade difusos acarretando em custos de agência e de incomplitude; 1) Necessidade de organização do quadro associativo e de estruturar sistema de informações visando transparência, participação e contribuições através de críticas e sugestões; m) Pode ocorrer a assimetria de informações tanto entre membros do Conselho de Administração quanto entre os dirigentes eleitos e os executivos contratados; n) Legislação específica voltada para o cooperativismo e para as cooperativas.

\section{ESTRUTURAS DE GOVERNANÇA}

A seguir serão apresentadas algumas estruturas organizacionais que poderão contribuir na construção da arquitetura organizacional das cooperativas. Inicialmente, na figura 1, apresenta-se a estrutura básica que retrata as principais áreas do sistema de governança nas organizações e, posteriormente, exemplos concretos da governança corporativa e da governança cooperativa.

Figura 1 - Estrutura básica de governança organizacional

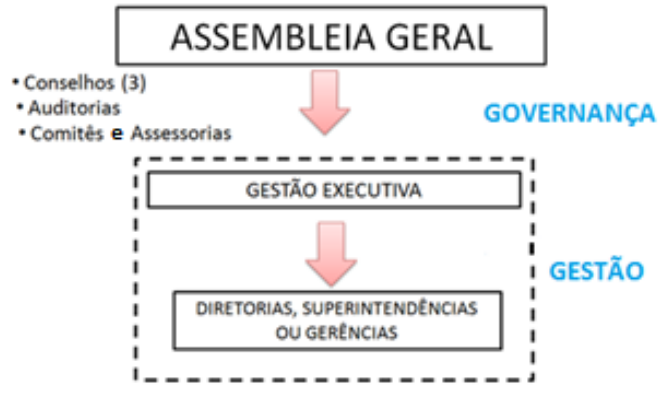

Fonte: Elaborado pelos autores

Esta estrutura apresenta de forma simples e inteligível, os elementos básicos da governança nas organizações salientando a segregação entre os órgãos superiores e gestão executiva das entidades. A figura 2 exemplifica a aplicação da estrutura básica numa organização.

Figura 2 - Governança nas organizações

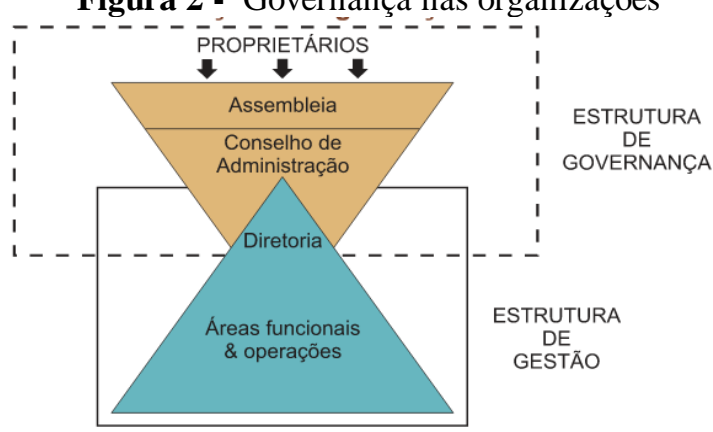

Fonte: Schiavini e Mello (2012, p.05) 
Esta estrutura contém os elementos do modelo 1, acrescidos de informações úteis para a maior parte das organizações. A figura
3 retrata a estrutura proposto pelo IBGC para cooperativas.

Figura 3 - Estrutura de governança para cooperativas segundo o IBGC

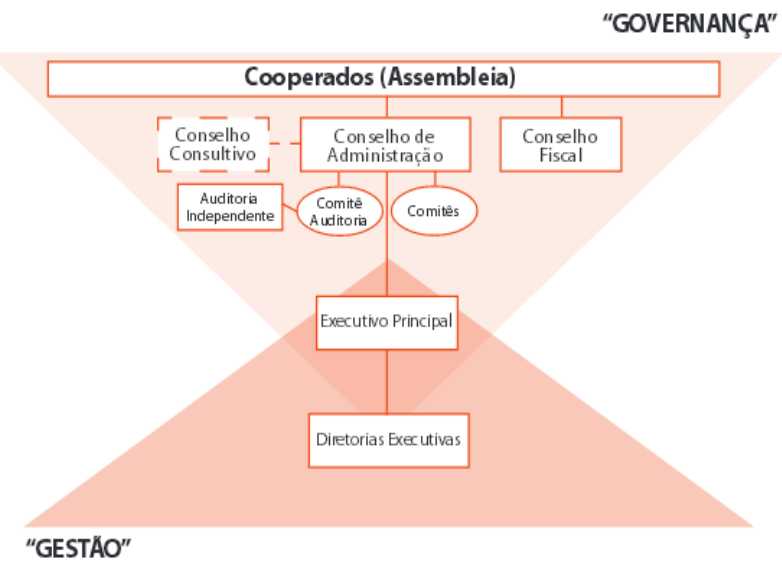

Fonte: IBGC, 2015

Esta estrutura, definida pelo IBGC, é associativismo. Menezes (2005) apresenta voltada para as cooperativas e pode ser outra forma de representar a estrutura de utilizada por outras entidades do governança em cooperativas.

Figura 4 - Estrutura alternativa de governança para cooperativas

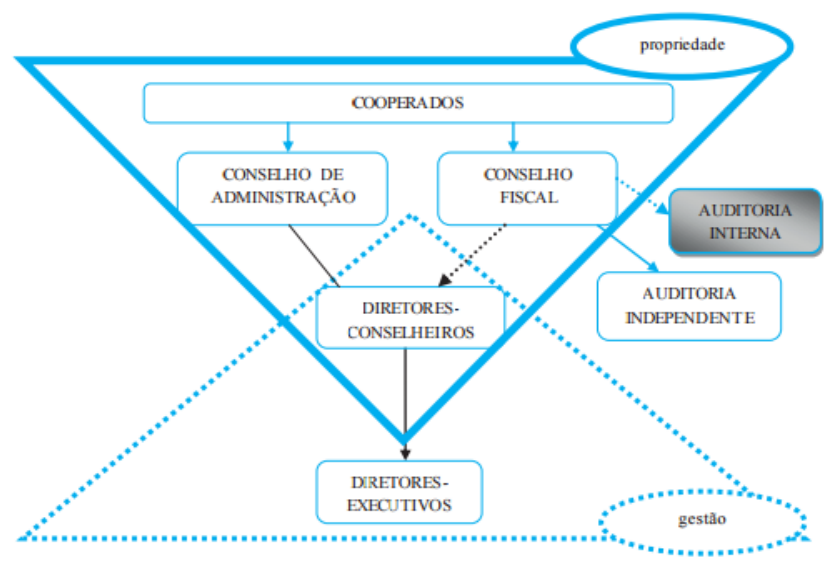

Fonte: Menezes, 2005

Esta estrutura também é direcionada para as cooperativas podendo ser utilizada por outras organizações associativistas. Por fim, a estrutura definida pelo Sistema OCB está espelhada na figura 5. 
Figura 5 - Estrutura de governança definida pela OCB

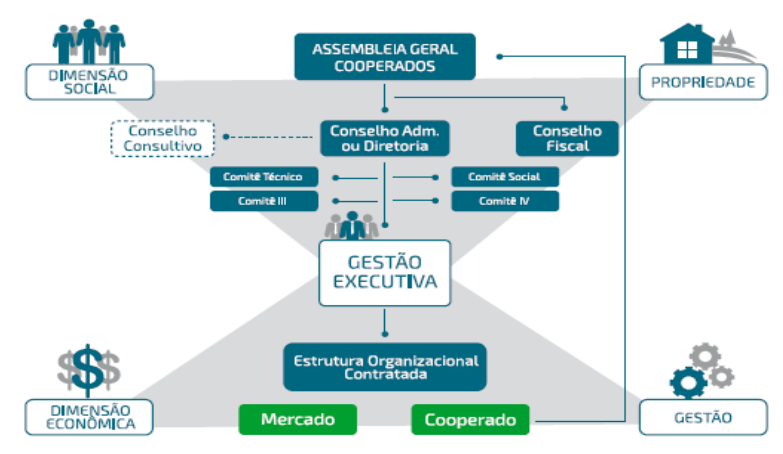

Fonte: Sistema OCB, 2013

11. PROPOSIÇÃO DE UMA ESTRATÉGIA PARA A ADOÇÃO DA GOVERNANÇA PELAS COOPERATIVAS

A estratégia pode ser operacionalizada através das etapas apresentadas a seguir, devidamente ajustadas para cada cooperativa: $\mathbf{1}^{\mathbf{a}}$ ) Diagnóstico elaborado com uso de técnicas administrativas; $\quad \mathbf{2}^{\text {a }}$ ) Qualificação da Cooperativa pela execução de estudos, benchmarking, debates, trabalhos, de comissões internas, eventos, negociação, consultorias voltadas para a governança; $3^{\text {a }}$ ) Formulação do Novo Projeto da Cooperativa em termos de estrutura, adequação dos ordenamentos, seleção de dirigentes, instâncias de poder e canais de comunicação; 4) Institucionalização do processo, após execução dos períodos de transição e de consolidação do processo; $\mathbf{5}^{\circ}$ ) Monitoramento da implantação através de indicadores e/ou outras técnicas de controle e avaliação.

\section{CONSIDERAÇÕES FINAIS}

Os resultados obtidos pelo estudo traduzem de forma concreta e explícita a compreensão e os posicionamentos de dez dirigentes de cooperativas eleitos ou contratados que integram quatro Ramos do cooperativismo.

Certamente, o diagnóstico expresso no trabalho disponibiliza valiosos subsídios que poderão servir de referência ou ponto de partida para estudos mais aprofundados e formulação de estratégias visando a implantação da governança nas cooperativas.

Não restam dúvidas de que a governança trará valiosas contribuições para o fortalecimento da fidelidade dos associados, tendo em vista que a fidelidade dos sócios com a sua cooperativa, segundo Ferreira (2016, p. 237) "é entendida como sendo a principal característica da cooperação e que a falta de fidelidade é um dos elementos centrais que alimentam um círculo vicioso que pode levar à extinção da organização cooperativa".

A governança contribui, também, para minimizar os seguintes principais problemas das cooperativas elencados por Pivoto (2015, p. 23): carona, horizonte, portfólio, controle e custos de influência.

A estratégia de adoção da governança sugerida no texto é fruto da compilação das dinâmicas utilizadas pelos dirigentes que já adotaram ou estão implantando a governança nas respectivas cooperativas. Portanto, constituem-se 
em valiosas referências para aquelas cooperativas que estão iniciando a sua implantação ou desejam obter subsídios fundamentados em práticas concretas já utilizadas por outras co-irmãs cooperativas.

De forma resumida, pode-se afirmar que não existe uma compreensão consolidada sobre governança nas cooperativas e que, ainda, não existe uma disposição fortemente deliberada para sua adoção nas mesmas. Neste sentido, sugere-se que haja maior estímulo e divulgação por parte do Sistema Cooperativista e das Universidades junto aos dirigentes de cooperativas, assegurando, assim, a motivação e a coragem que viabilizem sua adoção, oportunizando melhores resultados para os cooperados e a comunidade das suas respectivas regiões.

Ressalta-se, finalmente, que os resultados buscados pelo estudo centralizam seu foco na identificação das "vozes e dos sentimentos" de dirigentes de cooperativas sobre governança cooperativa.

\section{REFERÊNCIAS}

BANCO CENTRAL DO BRASIL. Governança cooperativa diretrizes para boas práticas de governança em cooperativas de crédito. 2008.

BANCO CENTRAL DO BRASIL. Diretrizes e mecanismos para fortalecimento da governança em cooperativas de crédito. 2009.

FERREIRA, G. M. V.; ARBAGE, A. P. Governança e sua relação com a fidelidade em cooperativa. Porto Alegre:Ed. Buqui, 2016.

GUIA DAS MELHORES PRÁTICAS DE GOVERNANÇA PARA COOPERATIVAS IBCG, 2015. Disponível em: http://www.ibgc.org.br/index.php/publicacoes/gui as Acesso em: 22 jun 2017.
MENEZES, Antônio. Nos rumos da cooperativa e do cooperativismo. Brasília, Confebrás, 2005.

NETO, Sigismundo Bialoskorki. Economia e gestão de organizações cooperativas. 2 . ed. São Paulo: Atlas, 2012.

PIVOTO, D. Governança cooperativa: os problemas dos direitos de propriedades difusos em cooperativas agropecuárias. 1.ed. Porto Alegre, RS: Buqui, 2015.

ROSSETTI, J. P.; ANDRADE, A. Governança corporativa: fundamentos, desenvolvimento e tendências. São Paulo: Atlas, 2014.

SESCOOP. Compêndio de boas práticas de gestão e governança. 2013/2014, 2015/2015 e 2017.

SISTEMA OCB. Manual de boas práticas de governança cooperativa, 2013.

SISTEMA OCB - site oficial, Disponível em: <http://www.ocb.org.br/> Acesso em: 20 jun 2017.

\footnotetext{
Adelar Francisco Baggio

Doutorando em Desenvolvimento Regional/UNIJUI, possui Graduação em Filosofia pela UNIJUI, PósGraduação na FGV e USP de São Paulo, mestrado em Economia Rural pela UFRGS. Atualmente é professor titular da Universidade Regional do Noroeste do Estado do Rio Grande do Sul -UNIJUI e Consultor Empresarial.
}

Martinho Luis Kelm
Possui graduação em Ciências Contábeis e
Administração pela UNIJU, mestrado em
Administração pela UFRGS e doutorado em
Engenharia de Produção pela UFSC. Professor do
Programa de Pós-Graduação em Desenvolvimento
Regional da UNIJUI


